\title{
Problemas-Proyectos Adaptativos y Creativos en la enseñanza de las ciencias. Descripción de la metodología y apreciación de los estudiantes involucrados
}

Adaptive and Creative Problem-Projects in the Teaching of Science. Description of the Methodology and Appreciation of the Students Involved

Problemes-Projectes Adaptatius i Creatius a l'ensenyament de les ciències. Descripció de la metodologia i apreciació dels estudiants involucrats

\author{
Miguel-Ángel Queiruga-Dios*® ${ }^{*}$, María-Consuelo Sáiz-Manzanares ${ }^{\circledR}$, Eduardo \\ Montero-García
}

Cómo citar: Queiruga-Dios, M. A.; Sáiz-Manzanares, M. C., y Montero-García, E. (2019).

Problemas-Proyectos

Adaptativos y Creativos en la enseñanza de las ciencias. Descripción de la metodología y apreciación de los estudiantes involucrados. Research in Education and Learning Innovation Archives, 23,1-23.

10.7203/realia.23.15567

Copyright: El/La Autor/a. Open Access: Este es un artículo de acceso abierto distribuido bajo los términos de la licencia Creative Commons AttributionNonCommercial-ShareAlike 4.0 International (CC BY-NC-SA 4.0)

Financiación: Proyecto KA219 del programa europeo Erasmus+ ATELIER for STEAM (2017-1-ES01KA219-038352). This communication reflects the views only of the author, and the Commission cannot be held responsible for any use which may be made of the information contained therein.
Universidad de Burgos, Burgos, España

*Autor para correspondencia: maqueiruga@ubu.es (Miguel-Ángel Queiruga-Dios)

Recibido: 15/05/2019 | Aceptado: 08/10/2019 | Publicado: 24/12/2019

RESUMEN: En este artículo se describe la metodología que se ha denominado Aprendizaje Basado en Problemas-Proyectos Adaptativos y Creativos (ABPac), utilizando como ejemplo algunos de los proyectos realizados en el centro concertado Jesús-María de Burgos durante diez años, entre 2006 y 2016, con alumnos de Secundaria Obligatoria y Bachillerato. Esta metodología sugiere una atención a la diversidad del alumnado considerando sus necesidades e inquietudes. Además, se analiza cuantitativamente el resultado de la encuesta de percepción realizada a 73 individuos involucrados en esta metodología cuando ya ha transcurrido un tiempo de madurez y se encuentran finalizando sus carreras o con estas ya finalizadas. Las conclusiones indican que esta metodología se muestra eficaz en los aspectos más relevantes que persigue: la adquisición de habilidades del siglo XXI (Scardamalia, Bransford, Kozma, y Quellmalz, 2012). Finalmente, se analiza el resultado de la encuesta realizada así como algunos testimonios recogidos.

PALABRAS CLAVE: ABP; enseñanza de las ciencias; habilidades; proyectos; problemas

ABSTRACT: This paper describes the methodology that has been called Adaptive and Creative Problem-Projects Based Learning (acPBL), using as an example some of the projects carried out in the Jesús-María concerted center in Burgos (Spain) for ten years, between 2006 and 2016, with students of Compulsory Secondary Education and Baccalaureate. This methodology suggests an attention to the diversity of the students considering their needs and concerns. In addition, the result of the perception survey carried out on 73 individuals involved in this methodology is quantitatively analyzed when a period of maturity has elapsed and they are finishing their degree studies or with these already completed. The conclusions indicate that this methodology is fully effective in the most relevant aspects pursued: the acquisition of 21st century skills (Scardamalia et al., 2012). Finally, the result of the survey conducted is analyzed as well as some collected testimonies.

KEYWORDS: PBL; teaching of sciences; skills; projects; problems

RESUM: En aquest article es descriu la metodologia que s'ha anomenat Aprenentatge Basat en Problemes-Projectes Adaptatius i Creatius (ABPac), utilitzant com a exemple alguns dels projec- 
tes realitzats en el centre concertat Jesús-María de Burgos durant deu anys, entre 2006 i 2016, amb alumnes de Secundària Obligatòria i Batxillerat. Aquesta metodologia suggereix una atenció a la diversitat de l'alumnat considerant les seves necessitats i inquietuds. A més, s'analitza quantitativament el resultat de l'enquesta de percepció realitzada a 73 individus involucrats en aquesta metodologia quan ja ha transcorregut un temps de maduresa i es troben finalitzant les seves carreres o amb aquestes ja finalitzades. Les conclusions indiquen que aquesta metodologia es mostra totalment eficaç en els aspectes més rellevants que persegueix: l'adquisició d'habilitats del segle XXI (Scardamalia et al., 2012). Finalment, s'analitza el resultat de l'enquesta realitzada així com alguns testimonis recollits.

PARAULES CLAU: ABP; ensenyament de les ciències; habilitats; projectes; problemes

\section{Notas de aplicación práctica}

\section{Qué se sabe sobre el tema}

- La metodología de Aprendizaje basado en proyectos/problemas facilita el desarrollo de habilidades en los estudiantes, sin embargo, puede resultar compleja la incorporación esta metodología al aula.

- Una de las creencias o justificaciones para no utilizar esta metodología es que no hay evidencias de su eficacia.

\section{Qué aporta este trabajo}

- La descripción de una metodología de Aprendizaje Basado en Proyectos y las pautas para su implementación en el aula, así como los resultados de su implementación.

- Evidencias de la apreciación de la mejora de las habilidades de los estudiantes que han participado en este tipo de aprendizaje por proyectos.

\section{Implicaciones para la práctica y/o política}

- La metodología descrita, ABPac, puede hacerse extensiva a todas las aulas, siendo necesaria la formación previa del profesorado para su implementación. Esta metodología, mejora el rendimiento de los estudiantes en el aprendizaje y contribuye al desarrollo de sus habilidades.

- En este artículo se sugieren algunas claves para propiciar el cambio metodológico en las aulas que repercutirán en una mejora de la práctica docente y un aumento de la eficacia de los procesos de enseñanza-aprendizaje.

\section{INTRODUCCIÓN}

\subsection{Definición de objetivos}

La introducción de metodologías activas en los procesos de enseñanza-aprendizaje de las ciencias requiere de un importante trabajo previo de diseño y organización por parte del docente (Badia y García, 2006; García, 2002). Si unimos esto a la complejidad de una evaluación en la que el profesor debe medir el grado de aprendizaje de contenidos conceptuales y de competencias (García-Martín y Pérez-Martínez, 2018; MorenoOlivos, 2010), además de la aparente lentitud a la que parece producirse, parecen justificadas las dudas que persisten entre el profesorado de ciencias sobre si los aprendizajes obtenidos con la utilización de metodologías activas son suficientes con respecto al esfuerzo dedicado a su adquisición. Por otro lado, incluso algunos de los estudiantes más brillantes, pueden preferir la seguridad y comodidad de una metodología clásica cuya evaluación se focaliza en la realización de una prueba escrita si este es el sistema de enseñanza-aprendizaje al que se han habituado (Vicente-Oliva y Andrés-Tirapo, 2007). Estas cuestiones hacen que en muchas ocasiones estas metodologías no se implementen en el aula, relegándose a temas de debate y modelos teóricos que se imparten en los cursos de formación del profesorado, o a lo sumo se realicen pequeñas experiencias de innovación didáctica. Si la implementación de metodologías activas 
ocasiona un esfuerzo adicional para profesores y estudiantes, ¿cómo puede el profesor tener la certeza de que el paso hacia el cambio metodológico es necesario?, ¿por qué forzar un cambio que parece contar con tantos detractores?

Muchos de los aprendizajes competenciales que se persigue que el alumno alcance con la implementación de las metodologías activas no se manifiestan inmediatamente, ya que su intención es preparar al estudiante para la vida profesional (Scardamalia, Bransford, Kozma, y Quellmalz, 2012, p. 246). Así como se critica la enseñanza tradicional porque el estudiante prepara unos contenidos memorísticamente y los olvida tras el examen, con las metodologías activas muchos de los aprendizajes se manifiestan a más largo plazo, ya que, su enfoque competencial, forma al estudiante con una capacidad propia de razonamiento y con un conjunto de habilidades que le permiten resolver situaciones cotidianas (Díaz-Barriga, 2006).

En este estudio pretendemos describir la metodología utilizada, a la que hemos querido nombrar como Aprendizaje Basado en Problemas-Proyectos Adaptativos y Creativos $^{1}$ (ABPac), por las particularidades que se describen más adelante, y que puede servir como modelo para su implementación en el aula o para su análisis crítico.

Otro de los objetivos de este artículo es conocer la percepción de los individuos que han trabajado con esta metodología activa cuando han transcurrido unos años desde que han dejado la etapa de Secundaria, de forma que el proceso de maduración les permite realizar un análisis crítico de sus aprendizajes durante etapas anteriores: cuando el estudiante está cursando su carrera, o la ha finalizado y se encuentra incorporado al mundo laboral o realizando sus estudios de doctorado. Es en ese momento cuando la persona es capaz de reflexionar sobre las habilidades desarrolladas durante su formación.

\subsection{Metodologías docentes de enseñanza-aprendizaje de las ciencias}

El término enseñanza tradicional de la ciencia suele referirse a aquella en la que el estudiante tiene un papel pasivo; es una enseñanza transmisiva centrada en contenidos conceptuales y que no tiene en cuenta la naturaleza de la ciencia ni cómo se aprende esta. Frente a esta enseñanza tradicional, surgen enfoques y metodologías que pretenden aproximarse a la forma en la que se construye la ciencia y hacer partícipe al alumno de esta construcción, haciendo que este se enfrente a los mismos problemas que se enfrenta el científico y del mismo modo obtenga soluciones (Pozo y GómezCrespo, 1998).

Las metodologías de enseñanza activa basadas en proyectos o problemas presentan algunas características en común:

- intervienen en la zona de desarrollo próximo del alumno (Vygotsky, 1979).

- el alumno es el protagonista del proceso de enseñanza-aprendizaje construyendo la ciencia y aplicándola, en la mayoría de los casos, a situaciones de la vida real (Rauscher, 2012), lo que puede llevar a aumentar el interés de los alumnos en las disciplinas científicas (Ribelles, Solbes, y Vilches, 1995). En las clases donde se implementan enfoques constructivistas los alumnos mejoran sus habilidades críticas y dan forma a su proceso de aprendizaje, además de contribuir al aumento de su autoestima al darles responsabilidad y valorar sus ideas (Gülbahar y Tinmaz, 2006).

- el profesor guía al alumno en el proceso de aprendizaje orientándole hacia el descubrimiento y a la construcción del conocimiento a través de una continua retroalimentación.

\footnotetext{
${ }^{1}$ Nota al lector: cuando se hable de proyectos en el contexto de las experiencias realizadas en este estudio se está refiriendo a la metodología que se describe en el presente artículo.
} 


\subsection{Enseñanza de las ciencias basada en la resolución de problemas}

En la enseñanza tradicional, habitualmente, el término resolución de problemas se refiere a la resolución de ejercicios cuantitativos similares a los que el profesor ha resuelto, a modo de ejemplo, tras explicar el tema correspondiente, y que son similares a los que aparecen al final del capítulo del libro de texto en uso. La creencia es que esto conduce a una comprensión conceptual, cuando muchas veces no es más que una sintetización y mecanización de una serie de pautas que, a través de una fórmula matemática, llevan a una solución numérica que generalmente el alumno es incapaz de interpretar. En realidad, la acepción de problema a la que se alude en el contexto de esta metodología de aprendizaje es "cualquier situación prevista o espontánea que produce, por un lado, cierto grado de incertidumbre y, por el otro, una conducta tendente a la búsqueda de su solución" (Perales-Palacios, 1993. p. 170). Los problemas deben ser factibles y realistas, focalizados en núcleos clave de las asignaturas que representen el trabajo continuado de todo el curso (García, 2002).

Algunos autores sitúan el origen del Aprendizaje Basado en Problemas en la Escuela de Medicina de la Universidad de McMaster de Canadá (Boud y Feletti, 1997; citado por Savery, 2015, p. 5). Pretende ser una propuesta educativa en la que el aprendizaje está centrado en el alumno, de forma que, además de que este aprendizaje sea significativo, permita desarrollar en él una serie de habilidades y competencias necesarias en el entorno profesional. Barrows (1986; citado por Campos, 2006, p. 1) define el Aprendizaje Basado en Problemas (ABP, o PBL, por sus siglas en inglés: Problem-based learning) como "un método de aprendizaje basado en el principio de usar problemas como punto de partida para la adquisición e integración de los nuevos conocimientos". Las características fundamentales de este modelo son:

- El aprendizaje está centrado en el alumno, guiado por un tutor. Es el alumno el que determina qué es lo que debe aprender y hasta qué punto debe llegar para ser capaz de resolver el problema.

- El aprendizaje se produce en pequeños grupos, que cambian de compañeros y tutor con cada nueva unidad curricular. Durante el aprendizaje auto-dirigido, los estudiantes discuten, revisan y debaten continuamente lo que han aprendido.

- El rol del profesor es el de facilitador o guía. A través de la formulación de preguntas logra que los alumnos se planteen cuestiones y elijan el mejor camino hacia la resolución del problema.

- El foco de organización del aprendizaje lo conforman los problemas. El profesor plantea un problema que representa un desafío para los estudiantes y los motiva para el aprendizaje. La organización necesaria para resolver el problema obliga a integrar múltiples disciplinas.

Para Paz-Penagos (2011) y Sáiz-Manzanares y Queiruga-Dios (2018), la resolución de problemas en la enseñanza de las ciencias favorece la metacognición, ya que da al estudiante la posibilidad de seleccionar, organizar y relacionar críticamente los contenidos a aprender, para lo que debe utilizar las estrategias de aprendizaje adecuadas; y además propicia la reflexión individual y grupal de las ideas. Esta metodología se establece como un enfoque alternativo a las prácticas tradicionales (García, 2002).

Aunque en líneas generales es difícil hacer una separación rigurosa entre aprendizaje basado en problemas y en proyectos, debido a los distintos enfoques que pueden realizarse, existen algunos elementos comunes que representan las fortalezas de estas metodologías y algunas diferencias (Rodríguez-Sandoval, Vargas-Solano, y LunaCortés, 2010). Ambas metodologías son constructivistas y centradas en el estudiante, en las que el profesor plantea un problema a resolver o un proyecto a realizar (GarcíaMartín y Pérez-Martínez, 2018) y donde los estudiantes trabajan en grupos o equipos 
guiados por el profesor. Mientras que en la metodología basada en proyectos se espera al final un producto tangible, como puede ser la construcción de un objeto o su diseño, en la metodología basada en problemas el resultado es una respuesta a la situación problemática (Reif, 2008).

Con las mismas siglas, ABP o PBL, algunos autores se refieren al aprendizaje basado en proyectos (Maldonado, 2008; Reverte-Bernabeu, Gallego-Sánchez, MolinaCarmona, y Satorre-Cuerda, 2007). Otros, sin embargo, matizan las diferencias entre Aprendizaje Basado en Problemas y Aprendizaje Basado en Proyectos, apareciendo, en ocasiones, modificadas las siglas de este último, ABPr (Domínguez, Carod, y Velilla, 2008; Rodríguez-Sandoval et al., 2010). Aunque existen distintos marcos que detallan las fases o etapas que sigue la metodología de Aprendizaje Basado en Proyectos, se describe a continuación el Gold Standard PBL (Larmer, Mergendoller, y Boss, 2015) promovido por ASCD (Association for Supervision and Curriculum Development), asociación sin ánimo de lucro fundada en 1943. Los beneficios de este método de aprendizaje, según los autores, son: key knowledge, understanding and success skills (conocimientos clave, comprensión y habilidades de éxito).

Esta metodología se define a través de la siguiente secuencia de fases:

- Problema o cuestión a responder (Challenging problem or question). Se trata de plantear un problema o cuestión para investigar y resolver. Puede ser un problema concreto o general, que tenga un grado medio de dificultad atendiendo a las competencias conceptuales y procedimentales de los alumnos.

- Indagación/investigación (Sustained inquiry). Esta fase de búsqueda de información o investigación es un proceso que puede llevar varios días dependiendo del problema planteado. En PBL, esta es una fase iterativa: cuando los estudiantes abordan el problema, se hacen preguntas que deben responderse buscando recursos, para, a continuación, hacerse preguntas más profundas. El proceso se repite hasta llegar a una solución satisfactoria. Los proyectos pueden incorporar diferentes fuentes de información, como libros, páginas web, además de recursos del mundo real como trabajo de campo, entrevistas con expertos, investigadores, empresas, etc. Los estudiantes pueden además realizar investigaciones de mercado analizando las necesidades de los usuarios.

- Autenticidad (Authenticity). El término autenticidad se refiere al hecho de que el aprendizaje o la tarea que se está realizando se está llevando a cabo como en el mundo real, en el sentido en que los estudiantes se enfrentan a la resolución de problemas como los que se resuelven en el mundo laboral y social, implicando procesos, herramientas y modos de hacer; "que los alumnos actúen como indagadores directos de la realidad" (Lacueva, Imbernón, y Llobera, 2002, p. 455). Esto aumenta la motivación por el aprendizaje (García, 2002).

- Voz y elección de los estudiantes (Student voice \& choice). Los estudiantes participan y controlan muchos de los aspectos del proyecto, desde las preguntas que generan, los recursos que utilizan para encontrar respuestas, las tareas y roles que asumen dentro del grupo, así como los productos que van a desarrollar. Incluso los estudiantes más avanzados pueden ir más allá y seleccionar el tema y la naturaleza del proyecto en sí.

- Reflexión (Reflection). Para Dewey (1995) el conocimiento se adquiere a partir de la reflexión sobre la experiencia. A lo largo del desarrollo del proyecto, tanto los alumnos como el profesor deben reflexionar sobre lo que están aprendiendo, cómo están aprendiendo y por qué están aprendiendo.

- Crítica y revisión (Critique \& revision). Es la fase de evaluación formativa. Los alumnos aprenden a dar y recibir retroalimentación constructiva de sus compañeros, lo que mejora los procesos y productos del proyecto. Para ello, utilizan 
rúbricas y protocolos de retroalimentación/crítica. También el profesor u otros agentes externos pueden participar en esta revisión. Los procesos de calibración y retroalimentación son componentes esenciales en el proceso de evaluación y aprendizaje (Sáiz-Manzanares y Montero, 2015).

- Producto público (Public product). Este aspecto es de gran importancia. El producto resultante del proceso de desarrollo del proyecto debe ser una cosa tangible o una solución a un proyecto real, en relación a lo comentado en el apartado de Autenticidad, y que estimula y motiva a los alumnos, que se preocupan más por la calidad de los productos. Cuando los estudiantes tienen que presentar su trabajo a un público más allá del aula, el nivel de desempeño aumenta. "La ciencia se produce, entre otras cosas, a partir del debate público, en el cual los científicos que desarrollan o usan teorías que compiten entre sí emplean la argumentación para posicionarse, defender sus ideas y oponerse a la de sus rivales, basándose en la concordancia entre los enunciados teóricos y las evidencias que tienen a su disposición" (Teixeira, Freire, y Greca, 2015). Además, mediante la creación de un producto, los estudiantes hacen tangible aquello que han aprendido, y el hecho de tener proyección más allá de las paredes del aula, ayuda a crear una comunidad de aprendizaje, en la que profesores y alumnos discuten lo que se aprende y cómo se aprende. Es una forma efectiva de comunicación con los padres, miembros de la comunidad educativa y el resto del mundo.

\section{DESARROLLO dE LA METOdOLOGÍA ABPAC}

Entre los años 2006 y 2016, se han desarrollado en el colegio Jesús-María de Burgos decenas de proyectos multidisciplinares, con alumnos de Secundaria Obligatoria y Bachillerato, siguiendo la metodología ABPac. A fin de mostrar al lector la validez de esta metodología, focalizamos la atención en aquellos que han sido galardonados en algún certamen nacional o internacional. Se han seleccionado algunos con estas características y en la Tabla 1 se muestran sus títulos a fin de que el lector pueda comprender la diversidad de temáticas tratadas.

Las condiciones generales sobre las que se han desarrollado estos proyectos han sido las siguientes:

- Libertad en la formación de los grupos. Los alumnos conforman los grupos como más adecuado les parezca. La justificación de este proceder es que, de alguna forma, el alumno puede agruparse con otros compañeros que tengan intereses similares o con los que ya está habituado a trabajar y a relacionarse. Con estos compañeros tendrá mayor confianza y facilidad para reunirse con el objetivo de avanzar el proyecto.

- Personalización del proyecto adaptándose al alumno, motivando una temática totalmente libre. La idea que subyace al dar una propuesta totalmente libre para comenzar el proyecto es que los propios alumnos puedan considerarlo como una oportunidad de desarrollar todas aquellas ideas que no encuentran un marco para su desarrollo en otros contextos. Al igual que en el apartado anterior, a lo largo de su vida académica el alumno desarrollará actividades, experiencias y proyectos más cerrados y dirigidos. Se trata de fomentar el desarrollo la creatividad, además de en la búsqueda de soluciones, en el planteamiento de problemas y necesidades a satisfacer. Por otro lado, la elección de una línea de investigación libre permitirá al alumno establecer conexiones entre la asignatura y la vida real.

- Seguimiento, tutorización, guía, retroalimentación. Es necesaria una continua retroalimentación profesor-equipos de forma que se resuelvan los problemas y dificultades que surjan, desde los primeros problemas de inicio (el alumno puede que 
Tabla 1. Ejemplos de algunos proyectos desarrollados con la metodología ABPac

\begin{tabular}{|c|c|c|}
\hline Título & Contenidos & Producto final/divulgación \\
\hline El aire que respiramos & $\begin{array}{l}\text { La atmósfera. Problemas } \\
\text { ambientales y soluciones. } \\
\text { Experiencias caseras. }\end{array}$ & $\begin{array}{l}\text { Página web, Animaciones } \\
\text { digitales }\end{array}$ \\
\hline Los minerales & $\begin{array}{l}\text { Rocas. Minerales. Clasificación. } \\
\text { Clave dicotómica. Juegos } \\
\text { interactivos. }\end{array}$ & $\begin{array}{l}\text { Página web, Animaciones } \\
\text { digitales }\end{array}$ \\
\hline Fotografía matemática & $\begin{array}{l}\text { Perspectiva matemática de nuestro } \\
\text { entorno cotidiano. Relaciones } \\
\text { geométricas. Otras geometrías: } \\
\text { fractales. Software matemático. }\end{array}$ & $\begin{array}{l}\text { Blog, Podcast, Vídeo, } \\
\text { Conferencias, Libro }\end{array}$ \\
\hline Multitema & $\begin{array}{l}\text { Revista de variedades con temas } \\
\text { de interés para los más jóvenes. } \\
\text { Entrevistas. }\end{array}$ & $\begin{array}{l}\text { Blog, Podcast, Vídeo, } \\
\text { Herramientas web 2.0: } \\
\text { presentaciones, } \\
\text { animaciones, etc. }\end{array}$ \\
\hline Electroquimicando & $\begin{array}{l}\text { Electroquímica. Experiencias } \\
\text { caseras. Juegos interactivos. }\end{array}$ & Página Web, Animaciones \\
\hline Leonardo Da Vinci & $\begin{array}{l}\text { Vida. Obra. Arte. Ciencia. } \\
\text { Construcción de inventos. }\end{array}$ & Web, Animaciones \\
\hline $\begin{array}{l}\text { El software libre, ¿realmente } \\
\text { alternativa? }\end{array}$ & $\begin{array}{l}\text { Software Libre. Aplicaciones. } \\
\text { Análisis comparativo. Estadísticas } \\
\text { de uso. }\end{array}$ & $\begin{array}{l}\text { Página Web, Vídeo, } \\
\text { Animaciones, Gráficos, } \\
\text { estadísticos online, Redes } \\
\text { Sociales Conferencias, } \\
\text { Libro }\end{array}$ \\
\hline Buscando vida ... el juego & $\begin{array}{l}\text { Astrobiología. El origen del } \\
\text { Universo. El origen de la vida. }\end{array}$ & $\begin{array}{l}\text { Póster, Blog, Juego de } \\
\text { mesa, Presentación en } \\
\text { ferias científicas }\end{array}$ \\
\hline 1, 2, 3, 4, .. iïHiggs!!! & $\begin{array}{l}\text { Partículas subatómicas. Masa y } \\
\text { energía. Fuerzas en la naturaleza. } \\
\text { Partículas elementales. El bosón } \\
\text { de Higgs. Cuestionarios. Juegos } \\
\text { interactivos. Entrevistas. }\end{array}$ & $\begin{array}{l}\text { Página Web, Blog, } \\
\text { Animaciones, Redes } \\
\text { Sociales, Libro }\end{array}$ \\
\hline Mars: weather and life, & $\begin{array}{l}\text { Sistema Solar. Condiciones de } \\
\text { Habitabilidad. Meteorología. } \\
\text { Análisis de datos meteorológicos. } \\
\text { El origen de la vida. Extremófilos. } \\
\text { Entrevistas. }\end{array}$ & $\begin{array}{l}\text { Blogs, Vídeo, Posters, } \\
\text { Conferencias, Artículos, } \\
\text { Redes Sociales, } \\
\text { Presentación en ferias } \\
\text { científicas }\end{array}$ \\
\hline $\begin{array}{l}\text { Mindstorms : detección de agua } \\
\text { en Marte }\end{array}$ & $\begin{array}{l}\text { Marte y vida. Aguan en Marte. } \\
\text { Robótica con Lego. }\end{array}$ & $\begin{array}{l}\text { Posters, Artículos, Redes } \\
\text { Sociales, Presentación en } \\
\text { ferias científicas }\end{array}$ \\
\hline $\begin{array}{l}2016 \text { Space. Conquistando el } \\
\text { espacio... }\end{array}$ & $\begin{array}{l}\text { Ciencia y ficción. Exoplanetas. } \\
\text { Instituciones. Exploración espacial. }\end{array}$ & $\begin{array}{l}\text { Blog, Animaciones, } \\
\text { Artículos, Redes Sociales, } \\
\text { Presentación en ferias Libro }\end{array}$ \\
\hline
\end{tabular}

no entienda lo que se le está pidiendo) hasta la orientación hacia nuevas fases de desarrollo del proyecto (subproblemas). El profesor toma el papel de director del grupo de investigación de la misma forma que un director gestiona los equipos en una institución científica.

Las características descritas nos llevan a utilizar las siglas ABPac, Aprendizaje Basado en Problemas-Proyectos Adaptativos y Creativos, orientados a las demandas, necesidades e intereses de los alumnos.

Resulta complejo definir una secuencia de fases o etapas para implementar la metodología $\mathrm{ABPac}$, ya que, como ocurre en el mundo real, uno traza un plan y unas líneas maestras a seguir, pero a medida que se va resolviendo el problema, van apareciendo 
nuevas líneas que nos sugieren mejores soluciones o soluciones más adecuadas en ese momento, por lo que podríamos hablar de un solapamiento de etapas y de un proceso de retroalimentación continua. Las fases con las que podemos representar esta metodología de planteamiento de problemas y búsqueda de soluciones, cuyo diagrama se muestra en la Figura 1, son las siguientes (Queiruga-Dios, 2017b):

- Fase de planteamiento y motivación (Approach and motivation). En esta fase se plantea a los alumnos: ¿Qué queréis investigar? (Queiruga-Dios, 2017a). Se motiva así a los estudiantes a que utilicen el contexto para desarrollar una investigación que, por falta de tiempo o recursos no han podido realizar o a que se enfrenten a una situación (problema) que deben resolver. Esto no resulta sencillo en general para la mayoría de los estudiantes, ya que suelen asociarlo en primera instancia al análisis o construcción de un producto tecnológico. No obstante, plantear problemas abiertos es un primer paso para el entrenamiento de la creatividad, una habilidad que pretende desarrollar esta metodología, y que constituye una diferencia respecto al Aprendizaje Basado en Proyectos o Problemas, en los que es el profesor el que especifica el problema a resolver (García-Martín y PérezMartínez, 2018). Las características iniciales de los proyectos deben ser mínimas, ya que el profesor se encargará de orientarlo hacia el currículum correspondiente. En esta fase, el profesor diseña un calendario orientativo con fechas de entregas y de evaluación del avance del proyecto.

- Formación de grupos (Make teams). El docente debe saber la importancia que tiene la formación de equipos de estudiantes (Pujolás, 2008a); de esto puede depender el éxito o fracaso de un proyecto. Pero también es importante que el propio estudiante sea capaz de experimentar y adquiera capacidad de analizar cómo ha afectado el equipo formado en el progreso del proyecto. Es por ello que, inicialmente, se da flexibilidad a la creación de equipos, proponiendo un tamaño orientativo (3 o 4 miembros), pero con posibilidad de justificar más o menos miembros. Cierto es que en el mundo real no siempre es posible elegir a los grupos de trabajo, pero también es cierto que grandes proyectos y empresas comienzan por un grupo de amigos o colaboradores previos. También es posible que, grupos con intereses similares deseen fusionarse; entonces se puede imponer la condición de que el producto final sea más elaborado. En cualquier caso, este es un momento para la flexibilidad y la creatividad, para no poner límites a la imaginación y al desarrollo del potencial del alumno. El profesor puede argumentar la necesidad del establecimiento de roles, empezando por un coordinador, cuya función principal sea la de engranar el funcionamiento del equipo y sirva de nexo con el profesor, y un secretario, que tome nota de las actas de reuniones y acuerdos. Las tareas que surjan en las siguientes fases definirán la aparición de nuevos roles y el reparto equitativo entre los miembros del equipo. El profesor revisará las actas u hojas de ruta, hablará con el coordinador y supervisará el reparto equitativo de tareas y la asunción de roles, teniendo en cuenta que el aprendizaje cooperativo es, en sí mismo, un contenido de aprendizaje (Pujolás, 2008b) y una de las habilidades que deben desarrollar los estudiantes (Scardamalia et al., 2012). La elaboración de documentos y hojas de ruta, en los que los alumnos reflejan el trabajo diario, los objetivos que persiguen, la temporalización, etc., así como Diagramas de Gannt (Domènech-Casal y RuizEspaña, 2017), puede facilitar el proceso de organización de los equipos. A modo de ejemplo se muestra en el Apéndice A el documento que pueden utilizar los equipos para elaborar su hoja de ruta.

- Fase de tormenta de ideas (Brainstorm). Los alumnos deben decidir qué fenómeno desean investigar o qué problema quieren resolver. Algunos equipos encon- 


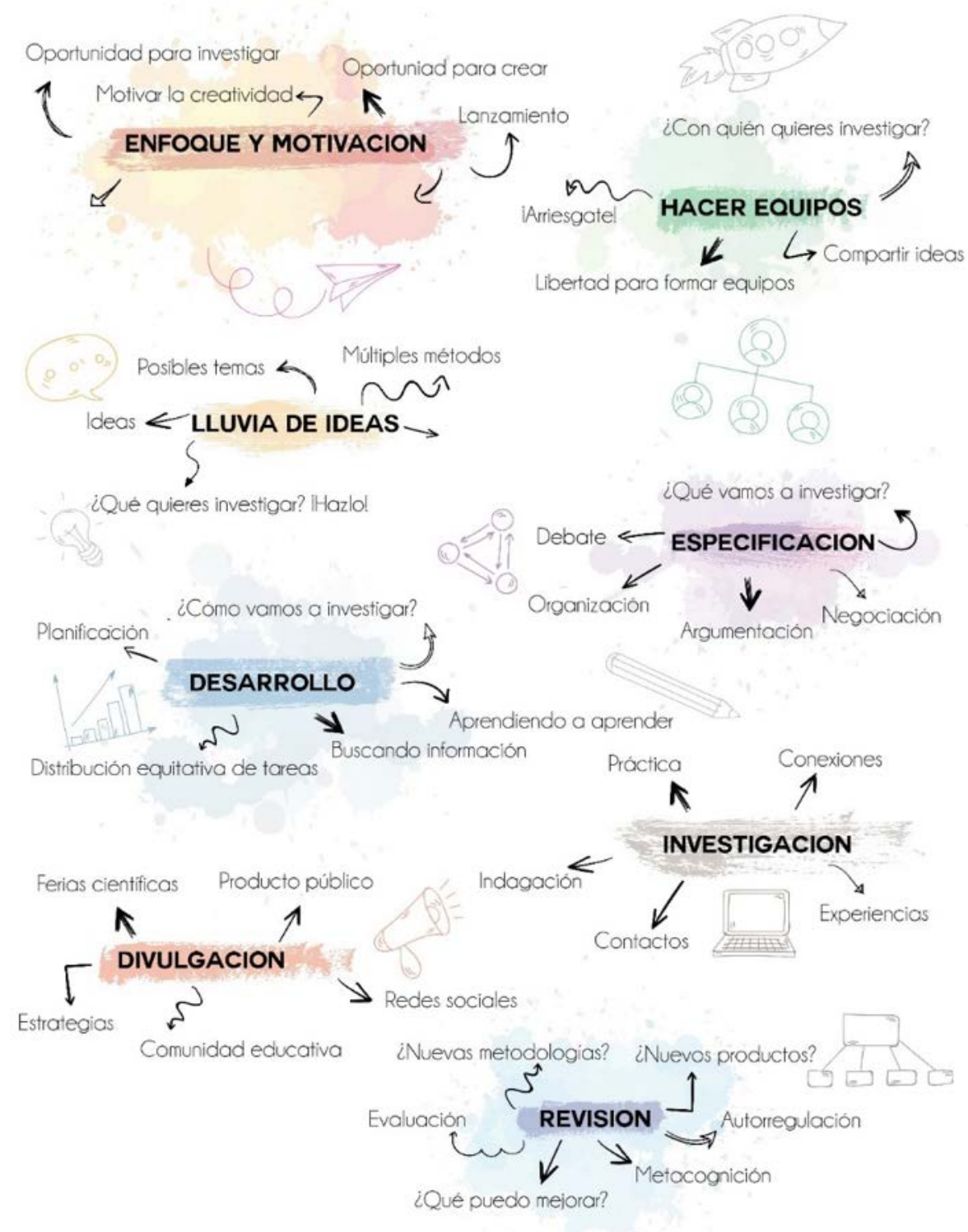

Figura 1. Diagrama de fases de un proceso ABPac. Elaboración propia

trarán una idea general en torno a la cual desarrollar su investigación, sin embargo, para otros resultará complicado, lo que refuerza la idea de la importancia de someter al estudiante a ejercicios en los que entre en juego la creatividad. A partir de estas ideas generales, el profesor orientará la investigación hacia contenidos específicos del currículum. Por ejemplo, si el estudiante desea investigar sobre robótica, el profesor puede concretarlo en diseñar un robot que explore Marte en busca de agua, y a su vez, conectar con la importancia del agua; o si el tema elegido por los estudiantes es el de las auroras boreales, el profesor puede guiar hacia aspectos más específicos: ¿qué fenómenos físicos hay involucrados?; y así llegar al estudio del espectro de emisión atómico o la interacción electromagnética. Tanto en esta fase como en la anterior, puede servir de referencia la información previa que el profesor puede tomar a partir de un sencillo cuestionario inicial recogido al comienzo del curso (Apéndice B).

- Fase de concreción (Specificatio). Llegados a este punto, los grupos ya están formados y cada equipo dispone de su problema a investigar. Cada equipo debe 
contar al profesor su idea inicial, respondiendo a las cuestiones de la Tabla 2. En función del tema elegido por el equipo, llegado el momento, el profesor suministrará materiales que permitan la realización de pequeñas prácticas, fuentes bibliográficas, etc. (se trata de establecer un diálogo con el alumno en el que el profesor facilita caminos cuando lo considera necesario o si el alumno se lo solicita). Todo el proceso debe documentarse con la toma de datos, fotografía y vídeo, elementos que posteriormente pueden ser utilizados para la difusión de la investigación.

Tabla 2. Qué, cómo, cuándo y dónde de la investigación

\begin{tabular}{|c|c|c|}
\hline ¿qué? & & Concreción del tópico \\
\hline ¿cómo? & vaic a invectiogr & Temporalización \\
\hline ¿cuándo? & & Estructura organizativa: distribución de tareas \\
\hline ¿dónde? & & Vías de comunicación \\
\hline
\end{tabular}

- Fase de desarrollo (Development). Durante esta fase los alumnos deberán documentarse sobre el tema de investigación. Inicialmente buscarán información en internet, a través de enciclopedias online o páginas que puedan encontrar a través de buscadores. No obstante, el profesor revisará las fuentes que utilizan sugiriendo a su vez nuevos recursos; para lo que será necesario que el profesor se actualice o se forme a la vez que busca apoyos en universidades e instituciones científicas. Cuando los alumnos adquieran el grado de expertos, deberán realizar visitas a instalaciones científicas o entrevistarse con científicos en persona, vía email o por videoconferencia.

- Fase de investigación (Investigation). Una vez que el alumno ya es experto en el tema que está tratando, tiene ya capacidad de plantearse nuevas preguntas y de buscar respuestas a estas nuevas preguntas, o bien, a medida que el equipo avanza en sus investigaciones, el profesor puede añadir nuevos enfoques o subproblemas.

- Fase de divulgación (Dissemination). Esta es una fase crucial en el desarrollo de cualquier proyecto de investigación. Desear divulgar la investigación realizada hace surgir nuevas cuestiones: ¿qué quiero mostrar a los demás?; esto induce una reflexión y revisión de la investigación llevada a cabo. Surge ahora un nuevo reto: ¿Cómo podemos dar a conocer nuestro proyecto entre los distintos públicos? Se deben analizar las posibles formas de difusión y diseño de productos, como podrían ser la creación de un programa de televisión, podcast, animaciones, posters, presentaciones, conferencias, etc. (Figura 2). Un importante ejercicio de creatividad.

- Fase de revisión (Review). Durante esta etapa se analizan los problemas surgidos, dificultades y mejoras que se pueden introducir (nuevas líneas de investigación, complementos, análisis del impacto producido en la divulgación, etc.). Se estudia también la posibilidad de continuar con el proyecto durante el siguiente curso.

La libertad en la elección del tema de investigación hace que estos sean muy diversos (como se muestra en la Tabla 1), pero un punto común es la utilización de las TIC en la búsqueda de información, elaboración, gestión e intercambio de documentos, comunicación, elaboración de presentaciones y elementos de divulgación (blogs, diseño de posters, etc.).

Los proyectos realizados siguiendo la metodología descrita se han desarrollado a lo largo de un curso académico, comenzando a principios de octubre, cuando los estudiantes ya están centrados en el curso y finalizando a mediados de mayo, antes de que 


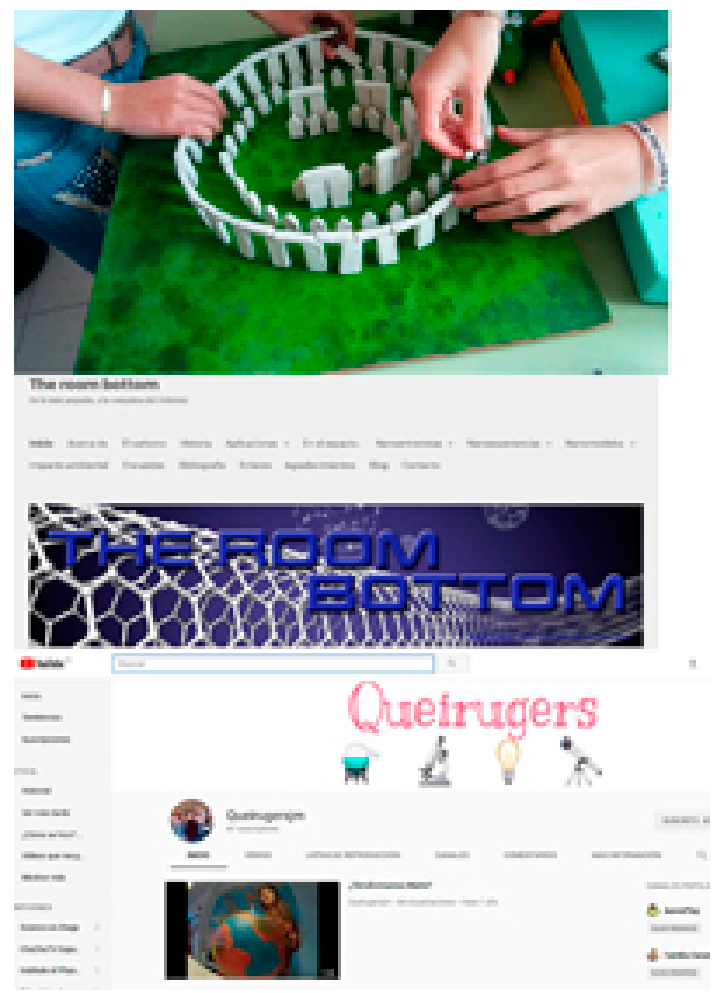

Figura 2. La creación de maquetas, blogs, canales de YouTube, etc., pone en marcha el desarrollo de nuevas competencias

los estudiantes comiencen las evaluaciones finales. Una temporalización orientativa, indicando las sesiones de trabajo presencial se muestra en la Figura 3. Los estudiantes realizan parte del trabajo en su casa y el profesor puede buscar momentos de interacción con los coordinadores o con los equipos para revisar la hoja de ruta y analizar el grado de avance del equipo fuera de las sesiones dedicadas específicamente a trabajar en el proyecto. Además, es interesante una comunicación fluida por correo electrónico.

\begin{tabular}{|c|c|c|c|c|c|c|c|c|}
\hline & Octubre & Noviembre & Diciembre & Enero & Febrero & Marzo & Abril & Mayo \\
\hline $\begin{array}{l}\text { Planteamiento } \\
\text { y motivación }\end{array}$ & \multirow{4}{*}{2} & & & & & & & \\
\hline $\begin{array}{l}\text { Formación de } \\
\text { grupos }\end{array}$ & & & & & & & & \\
\hline $\begin{array}{l}\text { Tormenta de } \\
\text { ideas }\end{array}$ & & & & & & & & \\
\hline Concreción & & & & & & & & \\
\hline Desarrollo & & \multicolumn{4}{|c|}{4} & & & \\
\hline Investigación & & & & \multicolumn{3}{|c|}{4} & & \\
\hline Divulgación & & & & & \multicolumn{4}{|c|}{4} \\
\hline Revisión & & & & & 1 & & & 1 \\
\hline
\end{tabular}

Figura 3. Temporalización de las fases del proyecto indicando el número de sesiones 


\section{DIFICULTADES DE LA IMPLEMENTACIÓN EN EL AULA}

Se describen a continuación algunas dificultades detectadas por los autores en la implementación de esta metodología en el aula.

En función de la experiencia previa de los estudiantes, puede ocurrir, como ya se ha indicado, que estos, acostumbrados a tareas muy dirigidas, se orienten hacia la construcción o descripción de objetos tecnológicos, o directamente a la realización de una presentación con diapositivas a partir de los contenidos encontrados en una página web. El profesor debe guiar a los estudiantes para que comprendan cuál es el objetivo de la propuesta de trabajo, si es necesario mostrando ejemplos y revisando con los estudiantes las pautas que deben seguir.

Conflictos menores pueden surgir en la formación de grupos, los mismos estudiantes suelen resolverlos sin necesidad de mediación del profesor, aunque es posible que en algún momento sea necesaria su intervención para favorecer la negociación. También puede ocurrir que, tras la tormenta de ideas, haya varios temas de interés entre los alumnos y, o bien se decanten por uno de los temas, o bien sea necesario reorganizar los equipos.

En cuanto al establecimiento de roles, es fundamental, durante las primeras sesiones, establecer el papel del alumno coordinador como conexión del profesor con el equipo. Después de esto, para evitar conflictos habituales, es imprescindible que en cada reunión los estudiantes redacten un acta con los aspectos tratados y que cada nueva tarea surgida se asigne a un responsable, además de determinar una temporalización (quién y cuándo), así quedará plasmado en la hoja de ruta qué estudiantes han realizado las tareas especificadas. Por ejemplo, si en una reunión deciden hacer un blog para divulgar su investigación, deberán especificar quién es el responsable, o quién es responsable de cada apartado del blog. Todos los miembros del equipo deben colaborar en cada tarea, pero tiene que haber un estudiante responsable de esa tarea. Estas consideraciones son cruciales para el buen desarrollo del proyecto. La retroalimentación debe ser un proceso continuo durante el cual el profesor comprueba el reparto equitativo de las tareas y el grado de desarrollo o avance en las mismas.

Además, puede ocurrir que algún equipo de alumnos considere que ya ha alcanzado el techo en el desarrollo de su proyecto. Es conveniente organizar alguna sesión en la que los estudiantes expongan su trabajo a sus compañeros y, a partir de la retroalimentación recibida por sus compañeros y por su profesor, encuentren nuevas líneas de desarrollo o mejora.

Aunque en general esta metodología es muy aceptada por los estudiantes, es posible que algún estudiante presente rechazo frente a la comodidad, hábito y seguridad de la enseñanza tradicional, por lo que se debe dar un peso importante en la evaluación a esta actividad.

Con respecto a la temporalización, es posible que la implementación de esta metodología en el aula no se ajuste estrictamente a la mostrada en la Figura 3. El docente debe ser consciente de todas las habilidades que los estudiantes están entrenando y de la importancia de que los estudiantes trabajen en una tarea en la que deben estar involucrados durante un curso completo, por lo que no debe considerarse el tiempo empleado como "tiempo perdido".

\section{ESTUDIO DE APRECIACIÓN}

Con el objetivo de valorar la apreciación de los individuos, transcurrido un tiempo, sobre cómo había influido la metodología ABPac en el desarrollo de sus competencias, se elaboró el cuestionario que se muestra en la Figura 4. Este cuestionario está formado por preguntas de respuesta cerrada y contestadas mediante una escala Likert de cuatro puntos desde muy en desacuerdo (1 punto) hasta muy de acuerdo (4 puntos). 
El cuestionario trata de recabar información sobre la apreciación en el desarrollo o mejora de las habilidades del siglo XXI (Scardamalia et al., 2012), que también pueden encontrarse entre las competencias transversales genéricas, como aparecen definidas en Aznar, Pujol, Sampere, y Rizo (2012) y la apreciación en el desarrollo de habilidades científicas (Blanco-López, España-Ramos, Franco-Mariscal, y Rodriguez-Mora, 2018; Franco-Mariscal, Blanco-López, y España-Ramos, 2014). Existe una correspondencia entre el cuestionario utilizado y el cuestionario validado CEMPA para medir la percepción de la efectividad del uso de Aprendizaje Basado en Proyectos, utilizado con estudiantes universitarios, elaborado por Carrasco-Gallego, Donoso-Anes, Duarte-Atoche, Hernández-Borreguero, y López-Gavira (2015). Esta correspondencia se muestra en el Apéndice $\mathrm{C}$.

Encuesta de apreciación sobre cómo me ha influido el haber trabajado en proyectos durante mi etapa de Secundaria. Valora desde muy en desacuerdo (1 punto) hasta muy de acuerdo (4 puntos).

Trabajar por proyectos ha mejorado la forma de:

\begin{tabular}{|l|l|l|l|l|}
\hline & 1 & 2 & 3 & 4 \\
\hline Resolver problemas/buscar soluciones adecuadas & & & & \\
\hline Tomar decisiones académicas & & & & \\
\hline Tomar decisiones personales & & & & \\
\hline Desenvolverme en el día a día & & & \\
\hline Hablar en público & & & \\
\hline Organizarme & & & & \\
\hline Relacionarme académicamente/laboralmente & & & \\
\hline Emprender nuevos proyectos & & & \\
\hline Mejorar la gestión de tiempos & & & & \\
\hline
\end{tabular}

Trabajar la metodología de proyectos me ha permitido:

\begin{tabular}{|c|c|c|c|}
\hline & 1 & 2 & 3 \\
\hline Profundizar en la metodología científica & & & \\
\hline Comprender qué es la ciencia & & & \\
\hline $\begin{array}{l}\text { Apreciar la ciencia como algo que se encuentra en mi } \\
\text { entorno }\end{array}$ & & & \\
\hline Desarrollar mis habilidades en el uso de las TIC & & & \\
\hline Mejorar la gestión de documentos científicos & & & \\
\hline Desarrollar mi capacidad de análisis & & & \\
\hline Interpretar datos & & & \\
\hline Discutir temas científicos & & & \\
\hline
\end{tabular}

Figura 4. Encuesta de apreciación

A lo largo del periodo de estudio (entre 2006 y 2016) se han desarrollado un total de 44 proyectos que podríamos encuadrar en esta metodología (Tabla 3). No se incluyen en esta a los alumnos o equipos que en años sucesivos han proseguido con su proyecto porque hayan querido profundizar en su investigación, aunque han sido guiados y orientados. En sucesivos cursos académicos esta metodología se ha generalizado en Secundaria con excelentes resultados, aunque todavía es pronto para hacer un estudio 
de apreciación similar al presentado en este artículo.

Tabla 3. Proyectos desarrollados por año académico

\begin{tabular}{lll}
\hline Año & $\begin{array}{l}\text { Número de proyectos } \\
\text { desarrollados }\end{array}$ & Número de alumnos involucrados \\
\hline $2006-2007$ & 4 & 20 \\
$2007-2008$ & 4 & 21 \\
$2008-2009$ & 5 & 23 \\
$2009-2010$ & 5 & 24 \\
$2010-2011$ & 5 & 26 \\
$2011-2012$ & 5 & 23 \\
$2012-2013$ & 4 & 22 \\
$2013-2014$ & 4 & 21 \\
$2014-2015$ & 4 & 25 \\
$2015-2016$ & 4 & 26 \\
Total & 44 & 231 \\
\hline
\end{tabular}

Se escogió una muestra aleatoria de 75 estudiantes involucrados en esta metodología, de los cuales, al cierre del estudio, habían contestado el cuestionario 73 individuos que, como se ha indicado, se encuentran realizando sus estudios universitarios o ya los han finalizado. No resulta complicado contactar con estos estudiantes a través de las redes sociales o por correo electrónico debido al vínculo que mantienen con el profesor, el centro o a través de otros antiguos compañeros.

La edad de los individuos participantes en este estudio oscila entre 19 y 30 años (Media=22,78; DT=3,06). De los individuos encuestados, más del 70\% está realizando o ha realizado estudios científico-tecnológicos. En la Tabla 4 se muestran los resultados obtenidos.

Se observa que todas las puntuaciones están muy próximas al valor máximo (4); siendo las respuestas con mayor puntuación algunas de las relacionadas con competencias científicas. Las respuestas que obtienen mayor puntuación, para los hombres, son: Desarrollar mis habilidades en el uso de las TIC (3.98), Mejorar la gestión de documentos científicos (3.85), Apreciar la ciencia como algo que se encuentra en mi entorno (3.78), que se consideran competencias científico-tecnológicas. Mientras, para las mujeres, las más valoradas son: Profundizar en la metodología científica (4), Apreciar la ciencia como algo que se encuentra en mi entorno (4), ambas competencias científicas, y, con la misma puntuación (3.94), Hablar en público, Organizarme y Relacionarme académicamente/laboralmente.

\section{DISCUSIÓN}

La necesidad creciente de implementar en el currículo el aprendizaje basado en proyectos y en indagación (National Academy of Sciences y of Medicine, 2011) conduce a que las intervenciones educativas estén enfocadas a la resolución de problemas, la autorregulación y las habilidades colaborativas (21st century skills) (Griffin et al., 2011; National Research Council, 2011, como se citan en OCDE 2013). En el documento A Framework for K-12 Science Education (National Research Council, 2012, p. 26), se matiza el hecho de que: "A pesar de que las prácticas utilizadas para desarrollar las teorías científicas y la forma que toman dichas teorías difieren de un dominio de la ciencia a otro, todas las ciencias tienen en común sus planteamientos basados en la indagación y la resolución de problemas". La enseñanza de la ciencia no puede concebirse como una transmisión de conceptos y tampoco puede hacerse extensiva la 
Tabla 4. Resultados de la encuesta de apreciación

\begin{tabular}{|c|c|c|c|c|c|c|}
\hline \multirow{2}{*}{ Trabajar por proyectos ha mejorado la forma de: } & \multicolumn{2}{|c|}{ Hombres } & \multicolumn{2}{|c|}{ Mujeres } & \multicolumn{2}{|l|}{ Total } \\
\hline & M & DT & M & DT & M & DT \\
\hline Resolver problemas/buscar soluciones adecuadas & 3.73 & 0.44 & 3.91 & 0.29 & 3.81 & 0.39 \\
\hline Tomar decisiones académicas & 3.73 & 0.44 & 3.81 & 0.39 & 3.77 & 0.42 \\
\hline Tomar decisiones personales & 3.71 & 0.45 & 3.81 & 0.39 & 3.75 & 0.43 \\
\hline Desenvolverme en el día a día & 3.73 & 0.44 & 3.84 & 0.36 & 3.78 & 0.41 \\
\hline Hablar en público & 3.73 & 0.44 & 3.94 & 0.24 & 3.82 & 0.38 \\
\hline Organizarme (secuenciar tareas, espacios, etc.) & 3.76 & 0.43 & 3.94 & 0.24 & 3.84 & 0.37 \\
\hline Relacionarme académicamente/laboralmente & 3.73 & 0.44 & 3.94 & 0.24 & 3.82 & 0.38 \\
\hline Emprender nuevos proyectos & 3.73 & 0.44 & 3.84 & 0.36 & 3.78 & 0.41 \\
\hline Mejorar la gestión de tiempos & 3.73 & 0.44 & 3.88 & 0.33 & 3.79 & 0.40 \\
\hline \multicolumn{7}{|l|}{ Trabajar la metodología de proyectos me ha permitido: } \\
\hline Profundizar en la metodología científica & 3.76 & 0.43 & 4.00 & 0.00 & 3.86 & 0.34 \\
\hline Comprender qué es la ciencia & 3.76 & 0.43 & 3.88 & 0.33 & 3.81 & 0.39 \\
\hline $\begin{array}{l}\text { Apreciar la ciencia como algo que se encuentra en mi } \\
\text { entorno }\end{array}$ & 3.78 & 0.41 & 4.00 & 0.00 & 3.88 & 0.33 \\
\hline Desarrollar mis habilidades en el uso de las TIC & 3.98 & 0.15 & 3.88 & 0.33 & 3.93 & 0.25 \\
\hline Mejorar la gestión de documentos científicos & 3.85 & 0.35 & 3.91 & 0.29 & 3.88 & 0.33 \\
\hline Desarrollar mi capacidad de análisis & 3.76 & 0.43 & 3.81 & 0.53 & 3.78 & 0.48 \\
\hline Interpretar datos & 3.73 & 0.44 & 3.75 & 0.61 & 3.74 & 0.52 \\
\hline Discutir temas científicos & 3.73 & 0.44 & 3.69 & 0.68 & 3.71 & 0.56 \\
\hline
\end{tabular}

transmisión de habilidades. En pro del éxito educativo, la enseñanza debe adaptarse al alumno individualmente. La diversidad debe ser entendida como algo natural e inherente a cada alumno, y la escuela debe ser inclusiva (UNESCO, 2005, p. 13), por lo que la enseñanza debe ser adaptativa, que fomente la autorregulación y las estructuras cooperativas del aprendizaje (Pujolás, 2008a). Además de los contenidos específicos de ciencia, el alumno debe aprender valores como convivencia, respeto y aceptación de la diversidad de compañeros, valores imprescindibles para una vida en sociedad. El aprendizaje activo, centrado en el alumno (Sandi-Urena, Cooper, y Stevens, 2012) y el aprendizaje colaborativo, donde se produce el aprendizaje por pares (Reif, 2008), son herramientas que propician la transmisión de estos valores. Por otro lado, tan importante como las actividades que se realizan es el CÓMO se realizan, y para ello es importante la preparación del profesor y la implicación (Angelini y García-Carbonell, 2015).

El análisis de los resultados de la encuesta de apreciación nos indica que los individuos encuestados consideran que la participación en este tipo de proyectos ha contribuido a desarrollar sus competencias generales así como sus competencias científicas. A pesar de que no hay diferencias significativas, la apreciación de las mujeres es que han desarrollado más sus competencias. Este desarrollo competencial concuerda con otras experiencias de implementación de ABP en Secundaria, en las que los estudiantes involucrados logran mejores resultados que con la enseñanza tradicional (DomènechCasal y Ruiz-España, 2017) y además se muestran más motivados e involucrados en el proceso de enseñanza aprendizaje (Benjumeda y Romero, 2017). Por lo tanto, si atendemos a la evaluación objetiva realizada por el profesor durante el proceso de enseñanza-aprendizaje y si, además, la apreciación de los estudiantes, transcurridos 
unos años, es que estas metodologías activas han contribuido al desarrollo de sus competencias, la conclusión es evidente: es un imperativo la implementación de este tipo de metodologías activas en el aula.

No obstante, los resultados obtenidos en este estudio contrastan con otros encontrados en el ámbito universitario (Aznar et al., 2012) en los que los estudiantes puntuaban muy bajo la apreciación sobre el desarrollo de habilidades comunicativas, habilidades para la vida y vida profesional (como la capacidad de liderazgo o la iniciativa) y la responsabilidad social. Es posible que esto dependa de cómo se ha implementado la metodología ABP o es posible que la percepción sobre el desarrollo de habilidades se produzca en el estudiante cuando ha transcurrido un tiempo de madurez. También podría ocurrir que las puntuaciones en la valoración del desarrollo de estas habilidades mejorasen (en concreto la capacidad de liderazgo, la iniciativa, y tal vez la responsabilidad social) si el alumno tomara más protagonismo en las elecciones de los temas en torno a los que desarrollar el proyecto de investigación o los equipos de trabajo, etc. Todos estos son aspectos a considerar en futuras investigaciones.

Los resultados de esta investigación están en consonancia con algunas opiniones recogidas previamente por los autores de algunos alumnos que en su día estuvieron involucrados en este tipo de proyectos ABPac, y que propiciaron este estudio posterior. Se reproducen a continuación algunas impresiones redactadas por los propios alumnos (Queiruga-Dios, 2015):

[...] todo este conocimiento que vamos adquiriendo elaborando pequeños trabajos nos será muy útil en nuestro futuro. [...] todo el proceso de creación y redacción del proyecto que uno esté llevando a cabo, es una de las formas más interesantes y eficientes de aprender.

La sensación de poder transmitir y publicar todo lo que has ido aprendiendo a lo largo de un proyecto de investigación es, en mi opinión, el paso más gratificante de todo el proceso de investigación. Creo por lo tanto que es muy importante incentivar a todos los estudiantes a que investiguen $[\ldots]$.

[...] si me preguntan por qué estudié una carrera de ciencias, contestaré que estos trabajos me acercaron a investigar, a querer saber más sobre aquello que nos rodea. Y si me preguntan por qué quiero ser profesora, contestaré que, gracias a mi experiencia, descubrí una manera nueva de aprender, en donde, el alumno se aleja de la pizarra y el pupitre, para acercarse a un aprendizaje más práctico y autodidacta, no solo adquiriendo conocimientos, sino también las capacidades para desarrollarlos.

[...] ese trabajo puso los cimientos para desarrollar determinadas aptitudes y actitudes que se pretenden con la educación: empezar a ser organizadas, buenas comunicadoras, creativas, innovadoras, constantes, disciplinadas y a ser muy tenaces, no nos dejaban rendirnos ante las dificultades o el desánimo, y sobre todo desarrollamos la paciencia. [...]. No éramos conscientes de todo lo que nos estaba aportando para el futuro. 
Como se aprecia en estas reflexiones, los estudiantes manifiestan que esta metodología de trabajo ha afectado a su forma de ver y acercarse a la ciencia, a su forma de aprender e incluso a las decisiones académicas que han tomado, considerando así que se han ejercitado y desarrollado muchas habilidades generales y científicas.

¿Cuáles parecen entonces, a simple vista, las condiciones mínimas para propiciar el cambio metodológico? Algunas de estas podrían ser:

- La reducción del número de alumnos por grupo. Una metodología inclusiva de calidad deberá partir del análisis de la realidad, fomentando la participación activa del alumnado e implicándoles en su proceso de enseñanza y aprendizaje, siendo protagonistas de su enseñanza (Avilés-Soler, Luque-De-La-Rosa, y HernándezGarre, 2015).

- Una revisión y puesta a punto de nuevas metodologías y escenarios. La metodología tradicional propicia que muchos alumnos adquieran un papel pasivo en el que terminan por acomodarse (García-Martín y Pérez-Martínez, 2018). La escuela inclusiva debe tener una flexibilidad que permita una adaptación de metodologías y escenarios al individuo (Queiruga-Dios, 2016). Además, este cambio debe empezar en las más tempranas edades, antes de que el alumno tome dicho papel pasivo en el proceso educativo.

- La actualización en metodologías de enseñanza-aprendizaje: cómo se aprende y qué se aprende. Se ha de alcanzar un nivel de profesionales versátiles en creatividad y autonomía para continuar su labor de formación permanente e innovar en materia educativa (Angelini y García-Carbonell, 2015), sabiendo que, como se muestra en este estudio y otros (Aznar et al., 2012; García-Martín y Pérez-Martínez, 2018; Queiruga-Dios, 2016), hay evidencias de efectividad en la adquisición de habilidades con respecto a la enseñanza tradicional.

- La reducción de la carga lectiva docente. Como consecuencia de lo apuntado en los apartados anteriores y los procesos de retroalimentación con los estudiantes, descritos ampliamente en este artículo, el docente debe dedicar gran parte de su tiempo de trabajo a la organización y diseño de grupos, de actividades, de proyectos, de escenarios, además de dedicar tiempo a su continua formación y por supuesto a la investigación y evaluación.

En futuras investigaciones, a pesar de la complejidad, sería interesante realizar una recopilación sistemática de datos que permitiera la realización de un estudio longitudinal a lo largo de la trayectoria del individuo. 


\section{A. APÉNDICE}

\section{Ejemplo de modelo de hoja de ruta}

\section{Nombre del equipo: \\ Coordinador.}

Secretario:

Otros componentes del equipo:

Título del proyecto:

Breve descripción o introducción:

Diseño del plan general inicial de actuación:

¿Qué voy a hacer? ¿Cómo lo voy a hacer? ¿Cómo nos repartimos las tareas? ¿Hasta dónde quiero llegar?

Temporalización

$1^{\text {a }}$ Reunión

Temas tratados, acuerdos, tareas que surgen, reparto de tareas, fecha para la siguiente reunión, etc..

$2^{\text {a }}$ Reunión

Temas tratados, acuerdos, tareas que surgen, reparto de tareas, fecha para la siguiente reunión, etc..

$3^{\mathrm{a}}$ Reunión

Temas tratados, acuerdos, tareas que surgen, reparto de tareas, fecha para la siguiente reunión, etc..

\section{$4^{\text {a }}$ Reunión}

Temas tratados, acuerdos, tareas que surgen, reparto de tareas, fecha para la sigulente reunión, etc...

Plan de acciones de divulgación

¿Cómo vas a dar a conocer el proyecto? ¿Alcance del proyecto?

Análisis de las dificultades surgidas a lo largo del proyecto y cómo se han superado. Indicad cómo replanteariais el proyecto (si tuvierais que volver a empezar...), cómo resolveriais los problemas surgidos, etc.

\section{Reflexión final}

¿Qué has aprendido? (No solo cosas que has aprendido sino nuevas formas de hacer) 
B. APÉNDICE

Ejemplo de cuestionario de intereses

¿Qué sabes acerca de la asignatura?

¿Tienes o has tenido dificultades con alguna asignatura?

En caso afirmativo, ¿cómo consigues superar estas dificultades?

¿Sueles llevar la asignatura al dia? Justifica

¿Qué quieres ser de mayor?

¿Sueles anticiparte a las posibles dificultades? Justifica

¿Te gustaria participar en algún proyecto de investigación? Justifica. En caso afirmativo, ¿tienes algún tema o idea en mente?

Algunas sugerencias:

_- Astrofísica

_- Astrobiologia

_- Robótica

_- Programación

__ Fotografía científica

_- Otros.... 


\section{APÉNDICE}

\section{Anexo III. Correspondencia del cuestionario con el CEMPA (Carrasco-Gallego et al., 2015)}

\begin{tabular}{lc}
\hline \multicolumn{1}{c}{ Trabajar por proyectos ha mejorado la forma de: } & Cuestionario CEMPA \\
\hline Resolver problemas/buscar soluciones adecuadas & 7 \\
Tomar decisiones académicas & 8 \\
Tomar decisiones personales & 8 \\
Desenvolverme en el día a día & 19,20 \\
Hablar en público & 12 \\
Organizarme & 9 \\
Relacionarme académicamente/laboralmente & $21,22,23,24$ \\
Emprender nuevos proyectos & 25 \\
Mejorar la gestión de tiempos & 6 \\
\hline \multicolumn{1}{c}{ Trabajar la metodología de proyectos me ha permitido: } & \\
\hline Profundizar en la metodología científica & 1,2 \\
Comprender qué es la ciencia & 1,2 \\
Apreciar la ciencia como algo que se encuentra en mi entorno & $1,2,5$ \\
Desarrollar mis habilidades en el uso de las TIC & 10 \\
Mejorar la gestión de documentos cientificos & 11 \\
Desarrollar mi capacidad de análisis & 11 \\
Interpretar datos & 11 \\
Discutir temas cientificos & 21,17 \\
\hline
\end{tabular}

\section{REFERENCIAS}

Angelini, M. L., y García-Carbonell, A. (2015). Percepciones sobre la integración de modelos pedagógicos en la formación del profesorado: La simulación y juego y el flipped classroom. Education in the Knowledge Society (EKS), 16(2). http://dx.doi.org/10.14201/eks20151621630

Avilés-Soler, B., Luque-De-La-Rosa, A., y Hernández-Garre, C. M. (2015). Estrategias metodológicas y organizativas para la mejora de la escuela inclusiva. Etic@net, 15(1), 1-1. Descargado de http://eticanet.org/revista/index.php/eticanet/article/view/67/62

Aznar, F., Pujol, M., Sampere, M., y Rizo, R. (2012). Adquisición de competencias mediante Aprendizaje Basado en Proyectos como metodología docente: valoración del alumnado. $X$ fornades de Xarxes d'Investigació en Docència Universitària: la participació i el compromís de la comunitat universitària, 1141-1153. Descargado de https://web.ua.es/es/ice/jornadas-redes-2012/documentos/posters/245822.pdf

Badia, A., y García, C. (2006). Incorporación de las TIC en la enseñanza y el aprendizaje basados en la elaboración colaborativa de proyectos. RUSC. Universities and Knowledge Society fournal, 3(2), 42-54. http://dx.doi.org/10.7238/rusc.v3i2.286

Benjumeda, F. J., y Romero, I. M. (2017). Ciudad Sostenible: un proyecto para integrar las materias científico-tecnológicas en Secundaria . Revista EUREKA sobre Enseñanza y Divulgación de las Ciencias, 14, 621-637. Descargado de https://revistas.uca.es/index.php/eureka/article/view/3157

Blanco-López, A., España-Ramos, E., Franco-Mariscal, A. J., y Rodriguez-Mora, F. (2018). Competencias y prácticas científicas en problemas de la vida diaria. Alambique: Didáctica de las ciencias experimentales, 92, 45-51.

Campos, F. S. (2006). El Aprendizaje Basado en Problemas como propuesta educativa para las disciplinas económicas y sociales apoyadas en el B-Learning. Revista Iberoamericana de 
Educación, 40(2), 3-3. Descargado de

https://dialnet.unirioja.es/servlet/articulo? codigo=2098520\&orden=80840\&info=link

Carrasco-Gallego, A., Donoso-Anes, J. A., Duarte-Atoche, T., Hernández-Borreguero, J. J., y

López-Gavira, R. (2015). Diseño y validación de un cuestionario que mide la percepción de efectividad del uso de metodologías de participación activa (CEMPA). Innovar, 25(58), 143-158. El caso del Aprendizaje Basado en Proyectos (ABPrj) en la docencia de la contabilidad. https://doi.org/10.15446/innovar.v25n58.52439

Council, N. R. (2012). A Framework for K-12 Science Education: Practices, Crosscutting Concepts, and Core Ideas. Washington, DC: The National Academies Press. https://doi.org/10.17226/13165

Dewey, J. (1995). Democracia y educación: una introducción a la filosofía de la educación. Madrid: Morata. (Reimpreso de Democracy and education: an introduction to the philosophy of education, (1916) por P. Monroe, Ed., 1916, New York: Macmillan)..

Díaz-Barriga, A. (2006). El enfoque de competencias en la educación: ¿Una alternativa o un disfraz de cambio? Perfiles educativos, 28, 7-36. Descargado de http://scielo.unam.mx/scielo.php?pid=S0185-26982006000100002\&script=sci_arttext

Domènech-Casal, J., y Ruiz-España, N. (2017). Mission to stars: un proyecto de investigación alrededor de la astronomía, las misiones espaciales y la investigación científica. Revista EUREKA sobre Enseñanza y Divulgación de las Ciencias, 14(1), 98-114. Descargado de https://revistas.uca.es/index.php/eureka/article/view/3008

Domínguez, J. A., Carod, E. S., y Velilla, M. J. (2008). Comparativa entre el aprendizaje basado en proyectos y el aprendizaje basado en problemas. II fornadas de innovación docente, tecnologıas de la información y de la comunicación e investigación educativa. Zaragoza. Descargado de https://cmapspublic2.ihmc.us/rid=1J9HKH72N-9B9GQG-T9F/ Informaci\{ \char"00F3\relax\}n\%20adjunta\%203.pdf II Jornadas de innovación docente. tecnologias de la información y de la comunicación e investigación educativa.

Franco-Mariscal, A. J., Blanco-López, A., y España-Ramos, E. (2014). El desarrollo de la competencia científica en una unidad didáctica sobre la salud bucodental. Diseño y análisis de tareas. Enseñanza de las Ciencias: revista de investigación y experiencias didácticas, 32(3), 649-667. http://dx.doi.org/10.5565/rev/ensciencias.1346

García, J. N. (2002). El aprendizaje basado en problemas: ilustración de un modelo de aplicaciones en psicopedagogía. Cultura y educación, 14(1), 65-79. Cultura y educación. https://doi.org/10.1174/113564002317348129

García-Martín, J., y Pérez-Martínez, J. E. (2018). Aprendizaje basado en proyectos: método para el diseño de actividades. Revista Tecnología, Ciencia y Educación(10). Descargado de https://tecnologia-ciencia-educacion.com/index.php/TCE/article/view/194/176 Revista Tecnología.

Gülbahar, Y., y Tinmaz, H. (2006). Implementing project-based learning and e-portfolio assessment in an undergraduate course. Fournal of Research on Technology in Education, 38(3), 309-327. https://doi.org/10.1080/15391523.2006.10782462

Lacueva, A., Imbernón, F., y Llobera, R. (2002). "El mundo de diferentes colores": tratando de enseñar conciencia planetaria a través de un proyecto de aula. Cultura y Educación, 14(4), 441-458. https://doi.org/10.1174/113564002762700907

Larmer, J., Mergendoller, J., y Boss, S. (2015). Setting the standard for project based learning: A proven approach to rigorous classroom instruction. Alexandria, VA: ASCD.

Maldonado, M. P. (2008). Aprendizaje basado en proyectos colaborativos. Una experiencia en educación superior. Revista de Educación Laurus, 14(28), 158-180. Descargado de http://www.redalyc.org/articulo.oa?id=76111716009 Recuperado de.

Moreno-Olivos, T. (2010). Reseña: Competencias en educación. Una mirada crítica. Revista mexicana de investigación educativa, 15(44), 289-297. Descargado de https://dialnet.unirioja.es/descarga/articulo/5404552.pdf

National Academy of Sciences, N. A. o. E., y of Medicine, I. (2011). Expanding Underrepresented Minority Participation: America's Science and Technology Talent at the 
Crossroads. Washington, DC: The National Academies Press.

https://doi.org/10.17226/12984

OCDE. (2013). Descargado de https://www.oecd.org/pisa/pisaproducts/

Draft\%20PISA\%202015\%20Collaborative\%20Problem\%20Solving\%20Framework\%20.pdf

Paz-Penagos, H. (2011). ¿Cómo desarrollar la metacognición en la educación superior mediante la resolución de problemas? . Ingeniería e investigación, 31(1), 213-223. Descargado de https://www.redalyc.org/pdf/643/64321170023.pdf Recuperado de.

Perales-Palacios, F. J. (1993). La resolución de problemas: una revisión estructurada. Enseñanza de las Ciencias, 11, 170-178. Descargado de http://www.raco.cat/index.php/Ensenanza/article/view/21188 Recuperado de.

Pozo, J. I., y Gómez-Crespo, M. A. (1998). Aprender y enseñar ciencia: del conocimiento cotidiano al conocimiento científico (and others, Ed.). Ediciones Morata.

Pujolás, P. (2008a). 9 ideas clave. El aprendizaje colaborativo (and others, Ed.). Barcelona: Graó.

Pujolás, P. (2008b). El aprendizaje cooperativo como recurso y como contenido. Aula de innovación educativa, 170, 37-41. Descargado de https://convivencia.files.wordpress.com/2008/11/au17008.pdf

Queiruga-Dios, M. A. (2015). Innovación en la enseñanza de las ciencias. A Coruña, España: Editorial Q.

Queiruga-Dios, M. A. (2016). Cómo convertir el aula en un escenario de aprendizaje significativo. En J. Gómez-Galán, E. López-Meneses, y A. H. M. Padilla (Eds.), Advances and Innovations in Educational Research (pp. 101-108). UMET Press.

Queiruga-Dios, M. A. (2017a). Adaptative \& Creative Problem Based Learning Science on Stage (Vol. 19). Archivo de vídeo.

Queiruga-Dios, M. A. (2017b). Adaptive and Creative Project-Problem Based Learning. En S. Csaba et al. (Eds.), Inventing the Future of Science Education. Debrecen, Hungary.

Rauscher, W. (2012). How project-based learning manifests itself in technology education textbooks. African fournal of Research in Mathematics, 16(1), 18-31. Science and Technology Education. https://doi.org/10.1080/10288457.2012.10740726

Reif, F. (2008). Applying cognitive science to education. Thinking and learning in scientific and other complex domains. Cambridge, MA: The MIT Press.

Reverte-Bernabeu, J. R., Gallego-Sánchez, A. J., Molina-Carmona, R., y Satorre-Cuerda, R. (2007). El Aprendizaje Basado en Proyectos como modelo docente. Experiencia interdisciplinar y herramientas Groupware. En and others (Ed.), XIII fornadas de Enseñanza Universitaria de la Informática. Descargado de http://hdl.handle.net/10045/1808

Ribelles, R., Solbes, J., y Vilches, A. (1995). Las interacciones CTS en la enseñanza de las ciencias. Análisis comparativo de la situación para la Física y Química y la Biología y Geología. Comunicación, Lenguaje y Educación, 7(4), 135-143. Descargado de http:// roderic.uv.es/bitstream/handle/10550/36653/098426.pdf? sequence=1\&isAllowed=y

Rodríguez-Sandoval, E., Vargas-Solano, E. M., y Luna-Cortés, J. (2010). Evaluación de la estrategia “aprendizaje basado en proyectos" . Educación y Educadores, 13(1), 143-158. 10.1590/S1414-40772010000100008

Sáiz-Manzanares, M. C., y Montero, E. (2015). Metacognition, Self-regulation and Assessment in Problem-Solving Processes at University. En A. Peña-Ayala (Series Ed.), Intelligent Systems. Metacognition: Fundaments, Applications, and Trends (Vol. 76, pp. 107-133). Switzerland: Springer. https://doi.org/10.1007/978-3-319-11062-2_5

Sáiz-Manzanares, M. C., y Queiruga-Dios, M. A. (2018). Evaluación de estrategias metacognitivas: aplicación de métodos online. Revista de Psicología y Educación, 13(1), 23-35. Revista de Psicología y Educación. 2018, V. 13, n. 1.

https://doi.org/10.23923/rpye2018.01.156

Sandi-Urena, S., Cooper, M., y Stevens, R. (2012). Effect of cooperative problem-based lab instruction on metacognition and problem-solving skills. fournal of Chemical 
Education, 89(6), 700-706. https://doi.org/10.1021/ed1011844

Savery, J. R. (2015). Overview of Problem-Based Learning: Definitions and Distintions. En A. Walker, H. Leary, C. Hmelo-Silver, y P. Ertmer (Eds.), Essential Readings in Problem-Based Learning: Exploring and Extending the Legacy of Howard S. Barrows (pp. 5-16). Purdue University Press. 10.7771/1541-5015.1002

Scardamalia, M., Bransford, J., Kozma, B., y Quellmalz, E. (2012). New assessments and environments for knowledge building. En P. Griffin, B. McGaw, y E. Care (Eds.), Assessment and teaching of 21st century skills (pp. 231-300). Dordrecht: Springer. https://doi.org/10.1007/978-94-007-2324-5_5

Teixeira, E. S., Freire, O., y Greca, I. M. (2015). La enseñanza de la gravitación universal de Newton orientada por la historia y filosofía de la ciencia: una propuesta didáctica con un enfoque en la argumentación. Enseñanza de las ciencias: revista de investigación y experiencias didácticas, 33(1), 205-223. Descargado de

https://www.raco.cat/index.php/Ensenanza/article/view/288579

UNESCO. (2005). Guidelines for inclusion Ensuring access to education for all (and others, Ed.). París, Francia: Autor. Descargado de http://www.ibe.unesco.org/sites/default/files/ Guidelines_for_Inclusion_UNESCO_2006.pdf

Vicente-Oliva, S., y Andrés-Tirapo, A. (2007). Resistencia de los alumnos al aprendizaje activo. En A. Herrera-Marteache, F. Serón-Arbeloa, y M. Sanagustín-Fons (Eds.), Innovación docente, tecnologías de la información y la Comunicación e Investigación Educativa en la Universidad de Zaragoza. Caminando hacia Europa. Universidad de Zaragoza.

Vygotsky, L. S. (1979). El desarrollo de los procesos psicológicos superiores (M. Cole, Ed.). Barcelona: Crítica. 